Zbigniew Grande, Mathematics Department, Pedagogical University, ul. Chodkiewicza 30, 85-064 Bydgoszcz, Poland

\title{
ON FUNCTIONS OF TWO VARIABLES EQUICONTINUOUS IN ONE VARIABLE
}

\author{
Abstract \\ The continuity of some functions of two variables equicontinuous in \\ one variable is considered.
}

Let $\mathbb{R}$ be the set of all reals and let $E$ denote $\mathbb{R}$ or $\mathbb{R} \times \mathbb{R}$. For $x \in E$ and for a positive real $r$ let $K(x, r)$ denote the open ball with center $x$ and radius $r$, i.e. $K(x, r)=\{t \in X:|t-x|<r\}$. Moreover, let $\mu_{e}(\mu)$ be the outer Lebesgue measure (the Lebesgue measure) in $E$.

Denote by

$$
\begin{aligned}
& d_{u}(A, x)=\limsup _{h \rightarrow 0^{+}} \mu_{e}(A \cap K(x, h)) / \mu(K(x, h)) \\
& \left(d_{l}(A, x)=\liminf _{h \rightarrow 0^{+}} \mu_{e}(A \cap K(x, h)) / \mu(K(x, h))\right)
\end{aligned}
$$

the upper (lower) outer density of a set $A \subset E$ at a point $x$. A point $x \in E$ is called a density point of a set $A \subset E$ if there exists a measurable (in the sense of Lebesgue) set $B \subset A$ such that $d_{l}(B, x)=1$. The family

$\mathcal{T}_{d}=\{A \subset E ; A$ is measurable and every point $x \in A$ is a density point of $A\}$ is a topology called the density topology $[1,2,3]$.

Moreover, let $\mathcal{T}_{e}$ denote the Euclidean topology in $E$.

Some examples of functions $f: E \rightarrow \mathbb{R}$ having continuous sections $f_{x}(t)=$ $f(x, t)$ and $f^{y}(t)=f(t, y), t \in \mathbb{R}$, whose sets of discontinuity points are of positive measure are well known [5]. On the other hand, if all sections $f_{x}$ of a function $f: E \rightarrow \mathbb{R}$ are equicontinuous at a point $y$ (i.e. for every positive real $\eta$ there is a positive real $\delta$ such that for every point $v$ with $|v-y|<\delta$

Key Words: $\mathcal{I}$-almost everywhere continuity, equicontinuity, separate continuity, density topology, functions of two variables

Mathematical Reviews subject classification: 26A15, 26B05, 54C08, 54C30

Received by the editors December 6, 1996 
and for every real $x$ we obtain $|f(x, v)-f(x, y)|<\eta)$ and if the section $f^{y}$ is continuous at a point $u$, then $f$ is continuous (as a function from $\left(E, \mathcal{T}_{e}\right.$ ) to $\left.\left(\mathbb{R}, \mathcal{T}_{e}\right)\right)$ at the point $(u, y)$. From this we obtain immediately the following remarks.

Remark 1. Suppose that all sections $f_{x}, x \in \mathbb{R}$, of a function $f: \mathbb{R}^{2} \rightarrow \mathbb{R}$ are equicontinuous at each point. Then $f$ is continuous at a point $(u, v)$ if and only if the section $f^{v}$ is continuous at $u$.

Remark 2. Let $\mathcal{I}$ and $\mathcal{J}$ be $\sigma$-ideals of subsets $\mathbb{R}$ and $\mathbb{R}^{2}$ respectively, such that every $F_{\sigma}$ set $A \subset \mathbb{R}^{2}$ having all sections $A^{y}=\{x ;(x, y) \in A\}, y \in \mathbb{R}$, belonging to $\mathcal{I}$ is in $\mathcal{J}$. If all sections $f_{x}, x \in \mathbb{R}$, of a function $f: \mathbb{R}^{2} \rightarrow \mathbb{R}$ are equicontinuous at each point and if all sections $f^{y}, y \in \mathbb{R}$, are $\mathcal{I}$-almost everywhere continuous (i.e. the sets $D\left(f^{y}\right)$ of all discontinuity points of $f^{y}$ belong to $\mathcal{I})$, then the function $f$ is $\mathcal{J}$-almost everywhere continuous.

As particular cases of the last remark we obtain the following.

Corollary 1. If all sections $f_{x}, x \in \mathbb{R}$, of a function $f: \mathbb{R}^{2} \rightarrow \mathbb{R}$ are equicontinuous at each point and if all sections $f^{y}, y \in \mathbb{R}$, are such that $\mu\left(D\left(f^{y}\right)\right)=0$ (all $D\left(f^{y}\right)$ are of the first category) then $\mu(D(f))=0(D(f)$ is of the first category).

We will show some stronger theorems.

Theorem 1. (a) Let $\mathcal{J}$ and $\mathcal{I}$ be some $\sigma$-ideals of subsets of $\mathbb{R}^{2}$ and of $\mathbb{R}$ respectively such that the vertical and horizontal projections of sets which are in $2^{\mathbb{R}^{2}} \backslash \mathcal{J}$ do not belong to $\mathcal{I}$. Suppose that there is a set $A \in \mathcal{I}$ such that all sections $f_{x}, x \in \mathbb{R}$, of a function $f: \mathbb{R}^{2} \rightarrow \mathbb{R}$ are equicontinuous at each point $y \in \mathbb{R} \backslash A$ and for every point $y$ the set $D\left(f^{y}\right)$ of all discontinuity points of the section $f^{y}$ is in $\mathcal{I}$. Then the set $D(f)$ of all discontinuity points of $f$ belongs to $\mathcal{J}$.

(b) Let $\mathcal{I}$ and $\mathcal{J}$ be some $\sigma$-ideals of subsets of $\mathbb{R}$ and of $\mathbb{R}^{2}$ respectively such that the vertical projections of sets which are in $2^{\mathbb{R}^{2}} \backslash \mathcal{J}$ do not belong to $\mathcal{I}$. Suppose that all sections $f_{x}, x \in \mathbb{R}$, of a function $f: \mathbb{R}^{2} \rightarrow \mathbb{R}$ are equicontinuous at every point and that all sections $f^{y}, y \in \mathbb{R}$, are $\mathcal{I}$-almost everywhere continuous (i.e. the sets $D\left(f^{y}\right)$ belong to $\left.\mathcal{I}\right)$. Then the set $D(f) \in \mathcal{J}$.

Proof. Suppose, to the contrary, that the set $D(f)$ of all discontinuity points of $f$ is not in $\mathcal{J}$. Consequently, there is a positive real $\eta$ such that the set

$$
B=\{(x, y) ; \operatorname{osc} f(x, y) \geq \eta\}
$$


is not in $\mathcal{J}$. Since all section $f_{x}, x \in \mathbb{R}$, are equicontinuous at each point $y \in \mathbb{R} \backslash A$, for every point $(x, y) \in B_{1}=B \backslash(\mathbb{R} \times A)$ there is an open interval $I(x, y)$ with rational endpoints such that $y \in I(x, y)$ and $|f(x, u)-f(x, y)|<$ $\eta / 4$ for all $u \in I(x, y)$ and $x \in \mathbb{R}$. The set $B_{1}$ is not in $\mathcal{J}$; so there is an open interval $I$ such that $B_{2}=\left\{(x, y) \in B_{1} ; I(x, y)=I\right\}$ is not in $\mathcal{J}$. Fix a point $(x, y) \in B_{2}$ and consider the section $f^{y}$. For every point $(t, u) \in B_{2}$ we have $|f(t, u)-f(t, y)|<\eta / 4$. But $B_{2} \subset B$, so for each point $(t, u) \in B_{2}$ there is a sequence of points $\left(v_{n}(t, u), w_{n}(t, u)\right)$ such that $w_{n}(t, u) \in I$,

$$
\left|f\left(v_{n}(t, u), w_{n}(t, u)\right)-f(t, u)\right|>3 \eta / 4
$$

for each positive integer $n$ and

$$
\lim _{n \rightarrow \infty}\left(v_{n}(t, u), w_{n}(t, u)\right)=(t, u) .
$$

For each positive integer $n$ and for each $(t, u) \in B_{2}$ we obtain

$$
\begin{gathered}
\left|f(t, y)-f\left(v_{n}(t, u), y\right)\right| \geq\left|f(t, u)-f\left(v_{n}(t, u), w_{n}(t, u)\right)\right|- \\
|f(t, u)-f(t, y)|-\left|f\left(v_{n}(t, u), w_{n}(t, u)\right)-f\left(v_{n}(t, u), y\right)\right|>3 \eta / 4-\eta / 4-\eta / 4=\eta / 4 .
\end{gathered}
$$

Since $\lim _{n \rightarrow \infty} v_{n}(t, u)=t$, the section $f^{y}$ is not continuous at any point of the set $F=\left\{t\right.$ : there is $u$ such that $\left.(t, u) \in B_{2}\right\}$ which is not in $\mathcal{I}$. So, the set $D\left(f^{y}\right)$ of discontinuity points of the section $f^{y}$ is not in $\mathcal{I}$, a contradiction. This contradiction finishes the proof of (a). The proof of the part (b) is analogous.

Corollary 2. If we suppose that $\mathcal{I}$ is the family of all subsets of $\mathbb{R}$ of measure zero (of the first category) [which are countable] and that $\mathcal{J}$ is the family of all subsets of $\mathbb{R}^{2}$ whose vertical projections belong to $\mathcal{I}$, then there is a set $A \in \mathcal{I}$ such that the set $D(f)$ of all discontinuity points of the function $f$ considered in Theorem $1(b)$ is contained in $A \times \mathbb{R}$.

Theorem 1 is not true for ideals. For example, if $I$ is the ideal of all finite subsets of $\mathbb{R}$ and if $\mathcal{J}$ is the ideal of all subsets $\mathbb{R}^{2}$ whose vertical projections are finite, then there is a function $f: \mathbb{R}^{2} \rightarrow \mathbb{R}$ having equicontinuous sections $f_{x}, x \in \mathbb{R}$, and such that for every $y \in \mathbb{R}$ the set $D\left(f^{y}\right)$ is finite and $D(f)$ is not in $\mathcal{J}$. For a construction of such a function we denote by $\mathbb{N}$ the set of positive integers, by $E(y)$ the greatest integer which is $\leq y$ and define

$$
f(x, y)= \begin{cases}\inf \{|y-v| ; v \in \mathbb{N}\} & \text { if } x \leq E(y) \\ 0 & \text { otherwise on } \mathbb{R}^{2}\end{cases}
$$


We will prove that Theorem $1(\mathrm{~b})$ is true for the ideals of nowhere dense subsets of $\mathbb{R}$ and $\mathbb{R}^{2}$, respectively. In the proof of the next theorem we will apply the following lemma.

Lemma 1. Suppose that the sections $f_{x}, x \in \mathbb{R}$, of a function $f: \mathbb{R}^{2} \rightarrow \mathbb{R}$ are equicontinuous at each point. If the function $f$ is not continuous at a point $(u, v)$ then there is an open interval I containing $v$ such that $f$ is not continuous at any point $(u, t)$, with $t \in I$.

Proof. Since $f$ is not continuous at the point $(u, v)$, there is a positive real $\eta$ such that $\operatorname{osc} f(u, v) \geq \eta$. From the equicontinuity of the sections $f_{x}, x \in \mathbb{R}$, at the point $v$ it follows that there is an open interval $I$ containing $v$ such that $|f(x, t)-f(x, v)|<\eta / 8$ for all $t \in I$ and $x \in \mathbb{R}$. There is a sequence of points $\left(u_{n}, v_{n}\right)$ such that $\lim _{n}\left(u_{n}, v_{n}\right)=(u, v)$ and $\left|f\left(u_{n}, v_{n}\right)-f(u, v)\right|>\eta / 2$ for $n=1,2, \ldots$. Since the section $f_{u}$ is continuous, we can assume that $u_{n} \neq u$ and $v_{n} \in I$ for $n=1,2, \ldots$. Observe that for each point $t \in I$ and for all $n=1,2, \ldots$ we have

$$
\begin{aligned}
\left|f\left(u_{n}, v_{n}\right)-f(u, t)\right| & \geq\left|f\left(u_{n}, v_{n}\right)-f(u, v)\right|-|f(u, v)-f(u, t)| \\
& >\eta / 2-\eta / 8=3 \eta / 8, \\
\left|f\left(u_{n}, v_{n}\right)-f\left(u_{n}, t\right)\right| & <\left|f\left(u_{n}, v_{n}\right)-f\left(u_{n}, v\right)\right|+\left|f\left(u_{n}, t\right)-f\left(u_{n}, v\right)\right| \\
& <\eta / 8+\eta / 8=\eta / 4
\end{aligned}
$$

and

$$
\begin{aligned}
\left|f\left(u_{n}, t\right)-f(u, t)\right| & \geq\left|f\left(u_{n}, v_{n}\right)-f(u, t)\right|-\left|f\left(u_{n}, v_{n}\right)-f\left(u_{n}, t\right)\right| \\
& >3 \eta / 8-\eta / 4=\eta / 8 .
\end{aligned}
$$

Since $\lim _{n} u_{n}=u$, we obtain that osc $f(u, t) \geq \eta / 8$ and $f$ is not continuous at the point $(u, t)$.

Theorem 2. If all sections $f_{x}, x \in \mathbb{R}$, of a function $f: \mathbb{R}^{2} \rightarrow \mathbb{R}$ are equicontinuous at each point and if for every $y \in \mathbb{R}$ the set $D\left(f^{y}\right)$ of all discontinuity points of the section $f^{y}$ is nowhere dense, then the set $D(f)$ is nowhere dense.

Proof. Suppose, by way of contradiction, that the set $D(f)$ is dense in an open rectangle $I \times J$, where $I, J$ are open intervals. Enumerate all open intervals with rational endpoints contained in $I$ in a sequence $I_{1}, \ldots, I_{n}, \ldots$. Denote by $D(f)$ the set of all discontinuity points of $f$. Let $\left(u_{1}, v_{1}\right) \in I_{1} \times J$ be a discontinuity point of $f$. By Lemma 1 there is a closed interval $J_{1} \subset J$ such that every point $\left(u_{1}, t\right)$ with $t \in J_{1}$ belongs to $D(f)$. Next, by induction in the $n^{t h}$ step $(n>1)$ we find a point $\left(u_{n}, v_{n}\right) \in\left(I_{n} \times \operatorname{int}\left(J_{n-1}\right)\right) \cap D(f)$ (int 
denotes the interior operation) and a closed interval $J_{n} \subset \operatorname{int}\left(J_{n-1}\right)$ such that for every point $t \in J_{n}$ the point $\left(u_{n}, t\right)$ is a discontinuity point of $f$. There is a point $w \in \bigcap_{n} J_{n}$. Since the section $f^{w}$ is not continuous at any point $u_{n}$, $n=1,2, \ldots$, and the set $\left\{u_{n} ; n \geq 1\right\}$ is dense in the open interval $I$, we obtain a contradiction. So, the set $D(f)$ is nowhere dense.

Denote by $\mathcal{I}_{G}$ and by $\mathcal{J}_{G}$ the ideals of all subsets of $\mathbb{R}$ and of $\mathbb{R}^{2}$ respectively, which are nowhere dense in every set belonging to $\mathcal{T}_{d}$.

Problem 1. Is Theorem 1(b) true for the ideals $\mathcal{I}_{G}$ and $\mathcal{J}_{G}$ ?

Theorem 3. Suppose that all sections $f_{x}, x \in \mathbb{R}$, of a function $f: \mathbb{R}^{2} \rightarrow \mathbb{R}$ are equicontinuous and for every $y \in \mathbb{R}$ the set $D\left(f^{y}\right) \in \mathcal{I}_{G}$. Then for all nonempty sets $K, L \in \mathcal{T}_{d}$ the set $D(f) \cap(K \times L)$ is nowhere dense in $K \times L$.

Proof. We can repeat the proof of Theorem 2. Suppose, by way of contradiction that there are linear nonempty sets $K, L \in \mathcal{T}_{d}$ such that the set $D(f) \cap(K \times L)$ is dense in $K \times L$. Let $I_{1}, \ldots, I_{n}, \ldots$ be an enumeration of all open intervals with rational endpoints for which $I_{n} \cap K \neq \emptyset, n=1,2, \ldots$. Let $\left(u_{1}, v_{1}\right) \in\left(I_{1} \cap K\right) \times L$ be a discontinuity point of the function $f$. By Lemma 1 there is an open interval $J_{1}$ containing $v_{1}$ such that every point $\left(u_{1}, t\right)$, where $t \in J_{1}$, belongs to $D(f)$. Next, in the $n^{t h}$ step $(n>1)$ we find a point $\left(u_{n}, v_{n}\right) \in\left(I_{n} \cap K\right) \times\left(J_{n-1} \cap L\right)$ belonging to $D(f)$ and a closed interval $J_{n} \subset \operatorname{int}\left(J_{n-1}\right)$ containing $v_{n}$ such that every point $\left(u_{n}, t\right)$, where $t \in J_{n}$, belongs to $D(f)$. Let $w \in \bigcap_{n} J_{n}$. Then the section $f^{w}$ is discontinuous at each point of the set $\left\{u_{n}, n=1,2, \ldots\right\}$, which is dense in $K \in \mathcal{T}_{d}$. So the set $D\left(f^{w}\right)$ is not in $\mathcal{I}_{G}$.

A function $f: E \rightarrow \mathbb{R}$ has property $\mathcal{A}$ at a point $x(f \in \mathcal{A}(x))$ ([4]) if for every positive $\eta$ and for every set $U \in \mathcal{T}_{d}$ such that $x \in U$ there is a nonempty open set $V$ such that $V \cap U \neq \emptyset, D(f) \cap U \cap V=\emptyset$ and $|f(t)-f(x)|<\eta$ for all points $t \in U \cap V$.

Evidently, if $f \in \mathcal{A}(x)$ for all $x \in E\left(=\mathbb{R}\right.$ or $\left.\mathbb{R}^{2}\right)$, then $D(f) \in \mathcal{I}_{G}$ or resp. $D(f) \in \mathcal{J}_{G}$.

Theorem 4. Suppose that all sections $f_{x}, x \in \mathbb{R}$, of a function $f: \mathbb{R}^{2} \rightarrow \mathbb{R}$ are equicontinuous and that for every point $(x, y) \in \mathbb{R}^{2}$ the relation $f^{y} \in \mathcal{A}(x)$. Then for every positive real $\eta$, for every point $(u, v)$ and for all nonempty linear sets $K, L \in \mathcal{T}_{d}$ with $(x, y) \in K \times L$ there is an open set $U$ such that $D(f) \cap(K \times L) \cap U \neq \emptyset,(K \times L) \cap U=\emptyset$ and $|f(s, t)-f(u, v)|<\eta$ for each point $(s, t) \in(K \times L) \cap U$.

Proof. Fix a positive real $\eta$, a point $(u, v)$ and sets $K, L \in \mathcal{T}_{d}$ with $(u, v) \in$ $K \times L$. Since $f^{v} \in \mathcal{A}(u)$, there is an open interval $I$ such that

$$
I \cap K \neq \emptyset, I \cap K \cap D\left(f^{v}\right)=\emptyset \text { and }|f(s, v)-f(u, v)|<\eta / 2
$$


for all points $s \in I \cap K$. From the equicontinuity of the sections $f_{x}, x \in \mathbb{R}$, it follows that there is an open interval $J$ containing $v$ such that

$$
|f(x, t)-f(x, v)|<\eta / 2
$$

for all points $x \in \mathbb{R}$ and $t \in J$. Evidently, $J \cap L \neq \emptyset$ and consequently,

$$
(I \cap K) \times(J \cap L)=(I \times J) \cap(K \times L) \neq \emptyset .
$$

For all points $(s, t) \in(I \cap K) \times(J \cap L)$ we obtain

$$
|f(s, t)-f(u, v)| \leq|f(s, t)-f(s, v)|+|f(s, v)-f(u, v)|<\eta / 2+\eta / 2=\eta .
$$

Since the sets $I \cap K$ and $J \cap L$ belong to $\mathcal{T}_{d}$, by Theorem 3 there is an open set $U$ such that

$$
U \cap((I \cap K) \times(J \cap L)) \neq \emptyset
$$

and

$$
D(f) \cap U \cap((I \cap K) \times(J \cap L))=\emptyset .
$$

Problem 2. Suppose that all sections $f_{x}, x \in \mathbb{R}$, of a function $f: \mathbb{R}^{2} \rightarrow \mathbb{R}$ are equicontinuous and that $f^{y} \in \mathcal{A}(x)$ for each point $(x, y) \in \mathbb{R}^{2}$. Is it true that $f \in \mathcal{A}(x, y)$ for each point $(x, y) \in \mathbb{R}^{2}$ ?

\section{References}

[1] A. M. Bruckner, Differentiation of Integrals, Amer. Math. Monthly, 78 Part II (1971), 1-51.

[2] A. M. Bruckner, Differentiation of real functions, Lectures Notes in Math. 659 (1978), Springer-Verlag.

[3] C. Goffman, C. J. Neugebauer and T. Nishiura, Density topology and approximate continuity, Duke Math. J. 28 (1961), 497-506.

[4] Z. Grande, On strong quasi-continuity of functions of two variables, Real Analysis Exch. 21 (1995-96), 236-243.

[5] R. Kershner, The continuity of functions of many variables, Trans. Amer. Math. Soc. 53 (1943), 83-100.

[6] T. Neubrunn, Quasi-continuity, Real Analysis Exch. 14 (1988-89), 259306. 